\title{
Improving Users' Consistency When Recalling Location Sharing Preferences
}

\author{
Jayant Venkatanathan ${ }^{1}$, Denzil Ferreira ${ }^{1}$, Michael Benisch ${ }^{2}$, Jialiu Lin $^{2}$, \\ Evangelos Karapanos ${ }^{1}$, Vassilis Kostakos ${ }^{1}$, Norman Sadeh ${ }^{2}$, and Eran Toch ${ }^{2}$ \\ ${ }^{1}$ Madeira Interactive Technologies Institute, University of Madeira \\ ${ }^{2}$ School of Computer Science, Carnegie Mellon University \\ \{vjayant, denzil.ferreira, e.karapanos, vk\}@m-iti.org \\ \{mbenisch, jialiul, sadeh, eran\}@cs.cmu.edu
}

\begin{abstract}
This paper presents a study of the effect of one instance of contextual cues, trajectory reminders, on the recollection of location sharing preferences elicited using a retrospective protocol. Trajectory reminders are user interface elements that indicate for a particular location of a person's trail across a city the locations visited before and after. The results of the study show that reminding users where they have been before and after a specific visited location can elicit more consistent responses in terms of stated location sharing preferences for that location visit. This paper argues that trajectory reminders are useful when collecting preference data with retrospective protocols because they can improve the quality of the collected data.
\end{abstract}

Keywords: Location sharing preferences, consistency, retrospective protocols.

\section{Introduction}

Location sharing applications are gaining wide adoption, with a number of commercial systems now available on the market, including Foursquare and Facebook Places. Such services are frequently used in the context of online social networks, whereby one's real-time location becomes yet another sharable aspect of one's online profile. With the increasing adoption of online location sharing services, understanding users' preferences and needs in terms of location sharing becomes crucial. Due to the nature of these services, it is methodologically difficult to elicit users' preferences in real time since this is likely to interrupt users' ongoing activities. Hence retrospection is a crucial methodological tool for eliciting users' preferences in this domain.

However, retrospective methods are susceptible to producing unreliable results. Due to their situated nature, location sharing preferences may depend on multiple contextual variables. Retrospective protocols may not reliably capture these characteristics and therefore elicit unreliable responses from users.

This paper presents a study that assesses the test-retest reliability of retrospective protocols, and introduces a technique for increasing the reliability of elicited responses in such protocols when collecting location sharing preferences. Grounded 
on experience-reconstruction theory [9], the technique entails the introduction of trajectory reminders in the data-collection GUI to help users recall episodic information that may be used to infer one's preferences for location sharing.

\section{Related Work}

There is an increasing amount of work on understanding users' location-privacy needs in ubiquitous and location-aware systems relying on techniques such as diary studies [1], interviews [5], surveys [8], scenarios [12] and lab and field observations [2]. A significant decision in understanding users' privacy needs in relation to location sharing is the methodology by which privacy preferences are elicited.

In attempting to elicit privacy preferences regarding location sharing, one could ask participants to provide an overall estimate of their preferences for a given location, such as one's workplace. However, these often-called global measures have been shown to underestimate the variability in perceptions and preferences as people often fail or incompletely reconstruct the particular context of each situation [10]. Robinson \& Clore [9] proposed a four-stage accessibility model of experiential information. At the heart of their model lies the distinction between episodic and semantic memory [13]. While episodic memory "is specific to a particular event from the past, semantic memory is not tied to any particular event but rather consists of certain generalizations (i.e. beliefs) that are rarely updated" [9]. In reconstructing one's emotions during an event, Robinson \& Clore's [9] model argues that he or she first attempts to recall contextual cues from episodic memory. When episodic memories become inaccessible (for instance because the event is located further in the past), people will shift to semantic memory.

The Experience Sampling Method (ESM) [4] attempts to avoid such retrospection and rationalization biases through probing the participant to report on ongoing behaviors and experiences. One of the drawbacks of ESM, however, is its laborintensive nature as it requires participants to interrupt their activities at numerous times within a day, while it may also miss important information when participants are not able to respond [15]. An alternative approach is the Day Reconstruction Method [6], a survey method that asks participants to recall in forward chronological order all experiences that took place in the previous day. Each experience is thus reconstructed within a temporal context of preceding and following ones. This is expected to cue more contextual information from episodic memory, and consequently recall the experienced emotion in a more valid and reliable manner. Kahneman et al. [6] showed that this method provides a surprisingly good approximation to Experience Sampling data, while providing the benefits of a retrospective method. Similarly Karapanos et al. [14] found that imposing a chronological order in the reconstruction of events resulted in an increase in the amount, the richness, and the test-retest reliability of recalled information.

In summary, literature suggests that if ESM is too intrusive then contextual cues should be used to help users reconstruct experiences from episodic memory. It follows that in asking participants to provide privacy-related preferences, a method that provides participants with contextual cues would elicit more reliable responses. To test this assumption in the context of location sharing privacy preferences, the 
study described next compares the reliability of privacy preferences when those are elicited with vs. without the help of contextual cues, which in this case are instantiated as trajectory hints. Specifically, the tested assumption is that reminding a participant where they have been before and after a visited location will cue more contextual information from the experience being measured, therefore resulting in more reliable and consistent recall of privacy preferences.

\section{Study}

A study was designed to test the hypothesis: Location Sharing privacy preferences elicited with the help of trajectory reminders will be more consistent than those elicited without the help of trajectory reminders.

A total of 20 participants were recruited with an average age of $28(\max =44, \min$ $=21$, median $=27$, s.d $=5.1$ ), through announcements on email lists, online forums, and fliers distributed across the campuses of the University of Madeira (Portugal) and University of Oulu (Finland). No reward was offered to participants. 9 participants (all male) were allocated to System A (no trajectory reminders) while 11 (8 male) were allocated to System B (with trajectory reminders). This difference in numbers in participants and genders across the two conditions was due to dropouts.

Each participant was given an Android smart-phone equipped with GPS logging software and was instructed to use this phone as their primary phone to ensure that they kept it with them at all times. During registration participants were also asked to list the names of five people from each of their family, close friends and colleagues. Each participant was asked to use the phone for a period of 4 days, spanning both weekdays and weekends. During this period the phone recorded each participant's location. The software interface allowed the participants the option to temporarily disable the logging software should they wish to do so. Participants were instructed to upload their location history data at the end of each day after which they were required to immediately do an online questionnaire task.

When the location history was uploaded, the set of "important locations" was selected from the uploaded data. Specifically, the chosen locations were those where the participants spent at least 5 minutes within a 50 meter radius. Subsequently, participants were taken through a series of questionnaire pages, where each page displayed an important location on the map along with the details of when the participant was there. Depending on the system to which participants were allocated, the location was displayed on a map with or without trajectory reminders. These reminders were arrows that indicated where the participant had been before and after visiting a location (Figure 1). All locations were displayed in chronological order.

As each important location was shown to participants, they were asked to recall how much information they would have liked to share about that specific location at that specific time with one specific person (chosen at random) from each of the 4 different recipient groups. The information was entered in a scale (1 to 5) with the following sharing options: (1) do not share, (2) region, (3) city, (4) neighborhood, (5) exact address. Besides entering their location sharing preferences, participants had the option to indicate that a location was completely wrong or invalid, although this option was never chosen. 

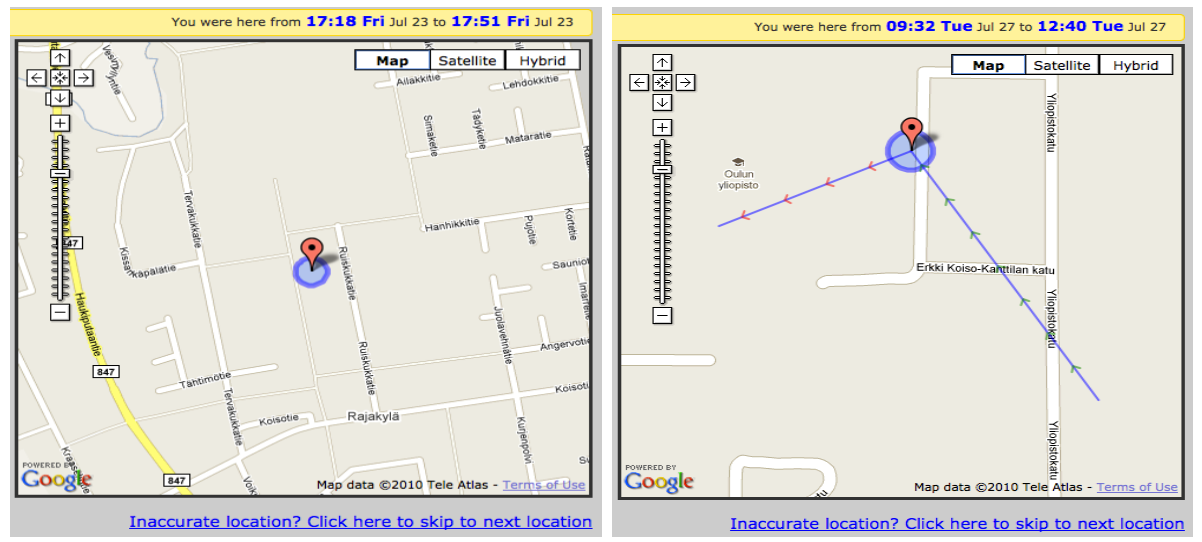

Fig. 1. The two versions of the map shown to participants. On the left is system A that shows no trajectory reminders. On the right is system $B$ that shows trajectory reminders, i.e. the locations visited before and after the location in question.

A week after returning the phones, participants were asked to access the online system and re-enter their preferences for each location they visited while they were carrying the phone. At this stage participants could not see their earlier responses. The previously recorded locations were shown with the same details and in the same order as the first time participants gave their preferences. Participants gave their preferences for all of their important locations in a single session in order to minimize the strain and reduce drop-out rates.

\section{Results}

The study was conducted between May and September 2010. A total of 441 distinct location visits were recorded, with each visit lasting on average 110 minutes. For each distinct visit to a location the following data was recorded: time and duration of visit, privacy preference (on a scale 1-5) for each of 4 possible recipient groups (family, close friends, colleagues, strangers), and the system being used (A or B). Of these, the independent variables were "System" and "Recepient_group". In addition, the difference in privacy preference was calculated by comparing the results from the two sets of questionnaires (the first was issued on the day of the visit to the location, the second was issued one week after the end of the study).

A chi-square test showed a significant effect of trajectory reminders (X-squared $=$ 43.5653, df $=8, \mathrm{p}<0.001)$ and recipient type $(\mathrm{X}$-squared $=116.038$, df $=24$, $\mathrm{p}<0.001)$ on the variation of privacy preferences. In terms of magnitude of the effect on consistency, the mean absolute difference in consistency for system A (no trajectory reminders) is 0.16 , and for system B (trajectory reminders) is 0.06 .

Figure 2 shows the mean variation in participants' responses, grouped by recipient type and system type. In these results a positive variation means that the follow-up response was more liberal (i.e. shared more information), a negative variation means that the follow-up response was more conservative (i.e. shared less information), while a smaller absolute variation means more consistent results. 


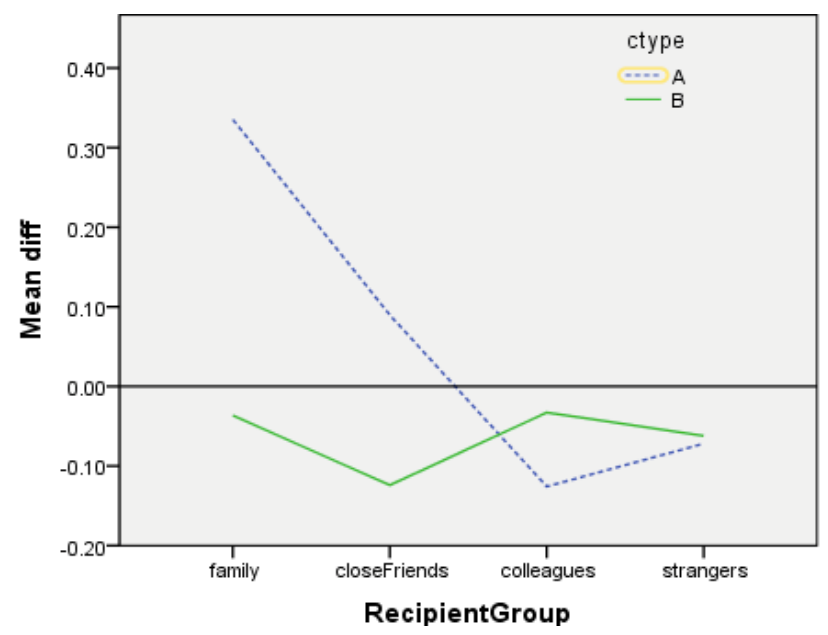

Fig. 2. Means difference in participants' responses (y-axis), grouped by recipient type (x-axis) and system type. Note that system A (dark blue) had no trajectory reminders, while system B (light green) had trajectory reminders.

\section{Discussion}

The results show that trajectory reminders (system B) increased the consistency of location sharing preferences elicited using a retrospective protocol, with users of System B being about twice more consistent in their responses. This suggests that trajectory reminders, and possibly other types of contextual cues, can significantly improve the reliability of location sharing retrospective protocols. It should be noted that both systems had a set of common reminders acting as contextual cues. These were, for example, the pinpointed location on a map that appeared in both systems, the names of the nearby streets, and the location of significant nearby landmarks. Hence, the difference in consistency across the two conditions is not due to the presence of reminders, but to the addition of an extra reminder in System B, namely trajectory. Therefore, it is expected that the data collection mechanism increased participants' consistency across both conditions, thus possibly masking the true effect of the trajectory reminders.

Orthogonal to the effect of trajectory reminders, the study showed a significant effect of recipient group on the consistency of participants' responses. Specifically, participants were most inconsistent in their preferences regarding family members, showing a tendency to increase the amount of information they chose to reveal to family members in the second questionnaire by about 0.13 points. On the other hand, participants' responses regarding the other recipient groups varied by up to 0.03 . This suggests that participants found it more challenging to reconstruct their experience and thus accurately recall their sharing preference in relation to close family members. An explanation for this finding is that participants tend to behave in predetermined manner when deciding how to share their location with non-family members, while not so for family members, thus leading to increased inconsistency. 
It should be noted that family members mean different things to different people, and family relationships can vary significantly. To minimize this discrepancy, the study required participants to give names of specific people to act as potential recipients in the questionnaire. This ensured that across the two questionnaires individual participants were asked questions about precisely the same potential recipients rather than, for example, "a family member". Clearly, however, the differences between participants' perception of family relationships may vary. Additionally, some participants noted that their family members live in a different city and hence had no reason to share their exact address with their family while they were in the city where this study was conducted. Their exact location within this city did not mean much to their family and hence did not practically reveal any useful information in addition to the city-level granularity.

\section{Implications}

An important implication for designing systems supported by the results is that the incorporation of trajectory reminders increases the consistency in participants' stated preferences. This implication is directly applicable to systems that employ mechanisms such as auditing and learning from the user [7]. Such systems, for example, allow users to examine location disclosure events that they or the system made, and indicate whether they are acceptable or not.

In addition to the implications for designing systems, the results presented here have important implications for designing studies. The methodological difficulty in eliciting location sharing preferences is that while techniques such as the experience sampling are too intrusive, retrospective protocols suffer from the fact that they may introduce some unreliability in the elicited preference data. The results of this study show that one mechanism by which such unreliability can be substantially reduced is by incorporating trajectory reminders when eliciting preference data.

The scope of this study, and its assessment of trajectory reminders, is strongly focused on the domain of location sharing applications. However, trajectory reminders may be themselves useful in studies unrelated to location sharing, but rather considering context-aware systems. Since location is an integral element of context, trajectory reminders may be used to help users reconstruct the context for a specific event for which they need to express a preference. Examples include studies that require users to recall preferences regarding, for example, a smartphones' behavior. It would be interesting to explore if the effect of trajectory reminders observed in this study would hold in a such a different context.

\section{Limitations}

It is important to keep in mind the context in which the results of the effect of trajectory reminders presented in this study were observed. The sample of participants comprised mainly of young students and staff (median age $=27$ ), mostly males, from a university, and care must be taken while trying to interpret the implications of these results to broader demographics. In addition, our study did not account for the 
differences in the recipient groups such as "family" between participants in the two conditions. Indeed, in an informal follow up interview one participant mentioned that his family members lived in a different city and hence it would have made no difference to his family whether he shared his exact location or just his city level location with them during the period of the study. Future work in understanding the effects of trajectory reminders must address these issues.

\section{Conclusion}

This paper demonstrates that trajectory reminders, which are a type of contextual cue, can help elicit more reliable responses from participants in retrospective protocols that collect location sharing preferences. The study shows that indicating the locations visited before and after a specific visited location can elicit more reliable location sharing preferences for that location visit. It is argued that trajectory reminders help participants reconstruct more accurately their experience of the location visit in question, and therefore provide more reliable stated preference responses.

Acknowledgements. This work is funded by the Portuguese Foundation for Science and Technology (FCT) grant CMU-PT/SE/0028/2008 (Web Security and Privacy).

\section{References}

1. Barkhuus, L.: Privacy in location-based services, concern vs. coolness. In: Mobile HCI 2004 Workshop: Location System Privacy and Control (2004)

2. Benisch, M., Kelley, P.G., Sadeh, N., Cranor, L.F.: Capturing location-privacy preferences: Quantifying accuracy and user-burden tradeoffs. Personal and Ubiquitous Computing, 1-16 (2010)

3. Eagle, N., Pentland, A.S., Lazer, D.: Inferring friendship network structure by using mobile phone data. Proceedings of the National Academy of Sciences 106((36), 1572415728 (2009)

4. Hektner, J.M., Schmidt, J.A., Csikszentmihalyi, M.: Experience Sampling Method: Measuring the Quality of Everyday Life. Sage Publications, Thousand Oaks (2007)

5. Hong, J.I., Landay, J.A.: An architecture for privacy-sensitive ubiquitous computing. In: MobiSys 2004, pp. 177-189 (2004)

6. Kahneman, D., Krueger, A.B., Schkade, D.A., Schwarz, N., Stone, A.A.: A Survey Method for Characterizing Daily Life Experience: The Day Reconstruction Method. Science 306(5702), 1776-1780 (2004)

7. Kelley, P.G., Hankes Drielsma, P., Sadeh, N., Cranor, L.F.: User-controllable learning of security and privacy policies. In: AISec 2008, pp. 11-18 (2008)

8. Khalil, A., Connelly, K.: Context-aware telephony: privacy preferences and sharing patterns. In: CSCW 2006, pp. 469-478 (2006)

9. Robinson, M.D., Clore, G.L.: Belief and feeling: Evidence for an accessibility model of emotional self-report. Psychological Bulletin 128(6), 934-960 (2002)

10. Schwarz, N., Kahneman, D., Xu, J., Belli, R., Stafford, F., Alwin, D., et al.: Global and episodic reports of hedonic experience. Calendar and Time Diary Methods in Life Course Research: Methods in Life Course Research, p. 157. Sage Pubns., Thousand Oaks (2008) 
11. Tsai, J.Y., Kelley, P., Drielsma, P., Cranor, L.F., Hong, J., Sadeh, N.: Who's viewed you?: the impact of feedback in a mobile location sharing application. In: CHI 2009, pp. 20032012 (2009)

12. Wagner, D., Lopez, M., Doria, A., Pavlyshak, I., Kostakos, V., Oakley, I., Spiliotopoulos, T.: Hide And Seek: Location Sharing Practices With Social Media. In: MobileHCI 2010, pp. 55-58.

13. Tulving, E.: Episodic Memory: From Mind to Brain. Annual Review of Psychology 53(1), 1-25 (2002)

14. Karapanos, E., Martens, J.-B., Hassenzahl, M.: Reconstructing Experiences through Sketching. Arxiv preprint, arXiv:0912.5343 (2009)

15. Hsieh, G., Li, I., Dey, A., Forlizzi, J., Hudson, S.E.: Using visualizations to increase compliance in experience sampling. In: Proceedings of the 10th International Conference on Ubiquitous Computing (2008) 